\title{
Amoeboid motion in confined geometry
}

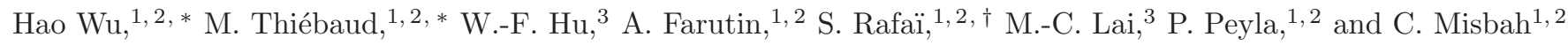 \\ ${ }^{1}$ Université Grenoble Alpes, LIPHY, F-38000 Grenoble, France \\ ${ }^{2}$ CNRS, LIPHY, F-38000 Grenoble, France \\ ${ }^{3}$ Department of Applied Mathematics, National Chiao Tung University, 1001 Ta Hsueh Road, Hsinchu 300, Taiwan
}

\begin{abstract}
Many eukaryotic cells undergo frequent shape changes (described as amoeboid motion) that enable them to move forward. We investigate the effect of confinement on a minimal model of amoeboid swimmer. Complex pictures emerge: (i) The swimmer's nature (i.e., either pusher or puller) can be modified by confinement, thus suggesting that this is not an intrinsic property of the swimmer. This swimming nature transition stems from intricate internal degrees of freedom of membrane deformation. (ii) The swimming speed might increase with increasing confinement before decreasing again for stronger confinements. (iii) A straight amoeoboid swimmer's trajectory in the channel can become unstable, and ample lateral excursions of the swimmer prevail. This happens for both pusher- and puller-type swimmers. For weak confinement, these excursions are symmetric, while they become asymmetric at stronger confinement, whereby the swimmer is located closer to one of the two walls. In this study, we combine numerical and theoretical analyses.
\end{abstract}

PACS numbers: 47.63.mh, 47.63.Gd, 47.15.G-,47.63.mf

Some unicellular micro-organisms move on solid surfaces or swim in liquids by deforming their body instead of using flagella or cilia-this is known as amoeboid motion. Algae such as Eutreptiella Gymnastica [1], amoebae such as dictyostelium discoideum 2, 3], but also leucocytes [2, 3] and even cancer cells [4] use this specific way of locomotion. This is a complex movement that recently incited several theoretical studies [5-12] since it is intimately linked to cell migration involved in several diseases. Some experimental results indicate that adhesion to a solid substratum is not a prerequisite for cells such as amoebae [2] to produce an amoeboid movement during cell migration and suggest that crawling close to a surface and swimming are similar processes. Recently, it was shown that integrin (a protein involved in adhesion process) should no longer be viewed as force transducers during locomotion but as switchable immobilizing anchors that slow down cells in the blood stream before transmigration. Indeed, leukocytes migrate by swimming in the absence of specific adhesive interactions with the extracellular environment [13].

When moving, all micro-organisms are sensitive to their environments. Most microswimmers can follow gradients of chemicals (chemotaxis), some microalgae can move toward light sources (phototaxis) [14] or orient themselves in the gravity field (gravitaxis) 15, some other bacteria move along adhesion gradients (haptotaxis) [16, 17], etc. Spatial confinement is another major environmental constraint which strongly influences the motion of micro-organisms. As a matter of fact, in the low-Reynolds number world, amoeboid motion generally occurs close to surfaces, in small capillaries or in extracellular matrices of biological tissues. Microorganisms swim through permeable boundaries, cell walls or micro-vasculature. Therefore, the effect of walls on motile microorganisms has been a topic of increasingly active research [18 27]. It has been calculated a long time ago by Katz [28] and more recently pointed out [21, 23, 25, 27, 29] that swimmers can take advantage of walls to increase their motility. Understanding the behavior of microswimmers in confinement can also pave the way to novel applications in microfluidic devices where properly shaped microstructures can interfere with swimming bacteria and guide, concentrate, and arrange populations of cells 30]. Living microswimmers show a large variety of swimming strategies [29] as do theoretical models aiming at describing their dynamics in confinement.

Felderhof [18] has shown that the speed of Taylor-like swimmer increases with confinement. Zhu et al. [21] used the squirmer model to show that (when only tangential surface motion is included) the velocity decreases with confinement and that a pusher crashes into the wall, a puller settles in a straight trajectory, and a neutral swimmer navigates. When including normal deformation they found an increase of velocity with confinement. Liu et al. [25] analyzed a helical flagellum in a tube and found that except for a small range of tube radii, the swimming speed, when the helix rotation rate is fixed, increases monotonically as the confinement becomes tighter. Acemoglu et al. 24] adopted a similar model but, besides the flagellum, they included a head and found a decrease of velocity with confinement. Bilbao et al. 222] treated numerically a model inspired by nematode locomotion and found that it navigates more efficiently and moves faster due to walls. Ledesma et al.23] reported on a dipolar swimmer in a rigid or elastic tube and found a speed enhancement due to walls.

Here, we investigate, by means of numerical and analytical modeling, the effect of confinement on the behavior of an amoeboid swimmer, which is a deformable object subjected to active forces along its inextensible 
membrane. Our model swimmer is found to reveal interesting features when confined between two walls. (i) We find that straight trajectories might be unstable, independently of the nature of the swimmer (pusher or puller). (ii) For weak confinement, the swimming speed can either increase or decrease depending on the confinement strength. For strongly confined regimes, the velocity decreases in all cases recalling previous results on different models. (iii) The confined environment is shown to induce a transition from one to another type of swimmer (i.e. puller or pusher). These behaviors are unique to amoeboid swimming (AS) and point to a nontrivial dynamics owing to the internal degrees of freedom that evolve in response to various constraints.

The model. Amoeboid swimming is modeled here by taking a one-dimensional (1D) closed and inextensible membrane, which encloses a two-dimensional (2D) liquid of certain viscosity $\eta$ and is suspended in another fluid taken to be of the same viscosity, for simplicity. The extra computational complexity of dealing with confined geometry restricts our study to $2 \mathrm{D}$, which draws already rich behaviors. The effective radius of the swimmer is $R_{0}=$ $\sqrt{A_{0} / \pi}$, where $A_{0}$ is the enclosed area. The swimmer has an excess normalized perimeter $\Gamma=L_{0} /\left(2 \pi R_{0}\right)-1$ ( $L_{0}$ is the perimeter) with respect to a circular shape ( $\Gamma=0$ corresponds to a circle, whereas large $\Gamma$ signifies a very deflated, and thus amply deformable, swimmer). The strength of confinement is defined as $C=2 R_{0} / W$, with $W$ the channel width. Bounding walls are parallel to the $x$ direction and $y$ denotes the orthogonal one.

A set of active forces is distributed on the membrane that reacts with tension forces to preserve the local arclength. The total force density is given by

$$
\mathbf{F}=F_{a} \mathbf{n}-\zeta c \mathbf{n}+\frac{\partial \zeta}{\partial s} \mathbf{t}
$$

where $F_{a} \mathbf{n}$ is the active force to be specified below (which we take to point along the normal $\mathbf{n}$ for simplicity), $\zeta$ is a Lagrange multiplier that enforces local membrane incompressibility, $c$ is the curvature, $\mathbf{t}$ is the unit tangent vector and $s$ is the arclength. We impose zero total force and torque. In its full generality the active force can be decomposed into a Fourier series $F_{a}(\alpha, t)=$ $\sum_{k=-k_{\max }}^{k=k_{\max }} F_{k}(t) e^{i k \alpha}$ with $\alpha=2 \pi s / L_{0}$. We first consider the case $k_{\max }=3$, so that we are left with two complex amplitudes $F_{2}$ and $F_{3}$. Other configurations of the forces have been explored as well (see below). We consider cyclic strokes represented by $F_{2}=F_{-2}=-A \cos (\omega t)$ and $F_{3}=F_{-3}=A \sin (\omega t)$, where $A$ is the force amplitude.

The Stokes equations with boundary conditions (force balance condition, continuity of the fluid velocity and membrane incompressibility) are solved using either the boundary integral method (BIM) 31 or the immersed boundary method (IBM) 32.

Besides $\Gamma$ and $C$, there is an additional dimensionless

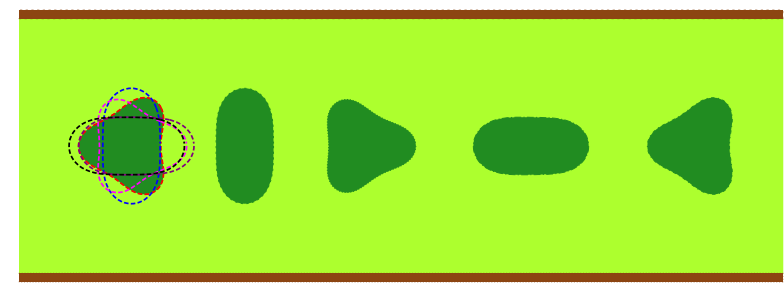

FIG. 1: (Color online) Snapshots of an axially moving swimmer over time $\left(W=6 R_{0}\right)$. The dashed profiles show a complete period $T_{s}$ of deformation and then a few shapes are represented over a time of the order of $75 T_{s} . \Gamma=0.085$.

number $S=A /(\omega \eta)$, which is the ratio between the time scale associated with swimming strokes $\left(T_{s}=2 \pi / \omega\right)$ and the time scale of fluid flow due to active force $\left(T_{c}=\eta / A\right)$. Here we take $S=10.0$ (the shape has enough time to respond to active forces) and explore the effects of $\Gamma$ and $C$. At a large distance from the swimmer, the velocity field is governed by $\sigma_{i j}=\oint F_{i} r_{j} d s$. Only the (dimensionless) stresslet $\Sigma=\left(\sigma_{x x}-\sigma_{y y}\right) /\left(\eta / T_{s}\right)$ enters the velocity field for symmetric swimmers. $\Sigma>0$ defines a pusher and $\Sigma<0$ defines a puller. The force distribution defined above is found to correspond to a pusher in the absence of walls. Below we will see how to monitor a puller or pusher and how the walls change the nature of the swimmer.

Results: Axially moving swimmers. We first consider an axially moving swimmer (AMS) (see Fig. 1). We consider only dimensionless quantities (unless otherwise stated). For example, $\bar{V}=V T_{c} / R_{0}$ will denote the magnitude of swimming speed. We find an optimal confinement for swimming velocity. Increasing $C$ enhances the speed of the swimmer until an optimal $C^{o}$, where the speed attains a maximum before it decreases. Around the optimal value $C^{o}$, low (high) viscous friction between the swimmer and the walls during the forward (recovery)

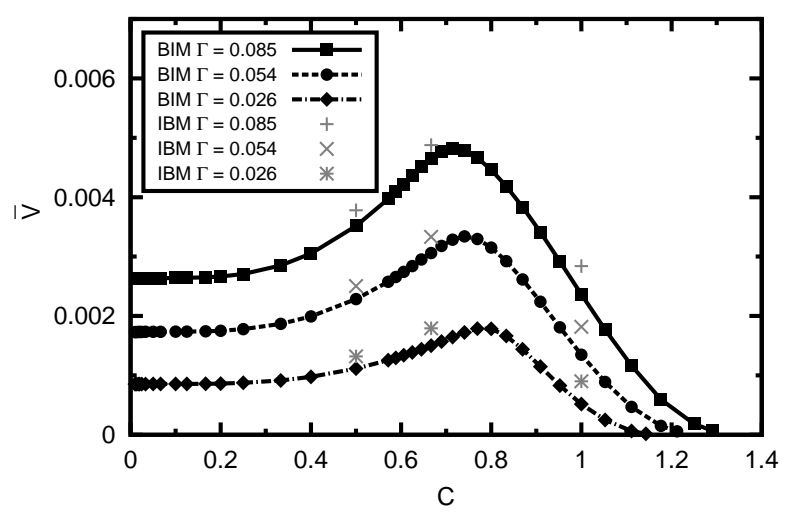

FIG. 2: Time-averaged velocity magnitudes (as a function of confinement $C$ ) of an axially moving swimmer for different $\Gamma$ values. 


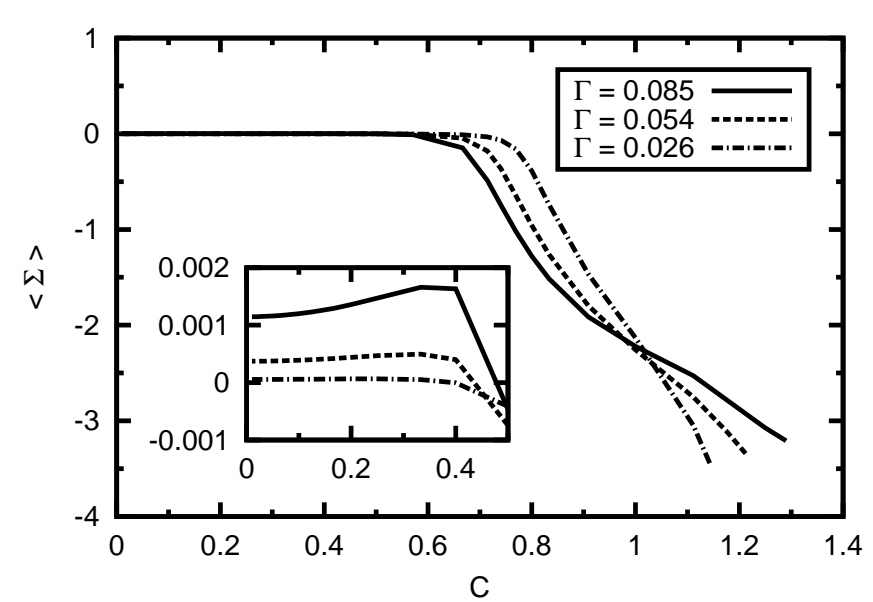

FIG. 3: Time-averaged $\langle\Sigma\rangle$ as a function of confinement $C$ showing the transition from pusher to puller.

phase of swimming promotes AMS speed. When the confinement is too strong, large amplitude deformations are frustrated resulting in a loss of speed. The velocity collapse at strong confinement was also reported for helical flagellum [24, 25] and is expected to occur for all swimmer models. Figure 2 shows the swimming velocity magnitude for different $\Gamma$ values. That the wall enhances motility seems to be a quite general fact, as reported in the literature [18, 19, 21 25]. However, we must stress that this is not a systematic tendency. Close inspection shows that at weak confinement velocity first decreases before increasing, as shown in Ref. [33].

Results: Swimmer nature evolution. The value of the dimensionless stresslet $\Sigma$ depends on the instantaneous swimmer configuration and its sign instructs us on the nature of the swimmer. We determine the average stresslet over a navigation cycle. An interesting result is the effect of confinement on the pusher/puller nature of the swimmer. For small $C(<0.5)$ the swimmer is found to behave as a pusher, while it behaves as a puller for larger $C(>0.5)$. Figure 3 shows the evolution of $\langle\Sigma\rangle$ as a function of confinement, where a transition from pusher to

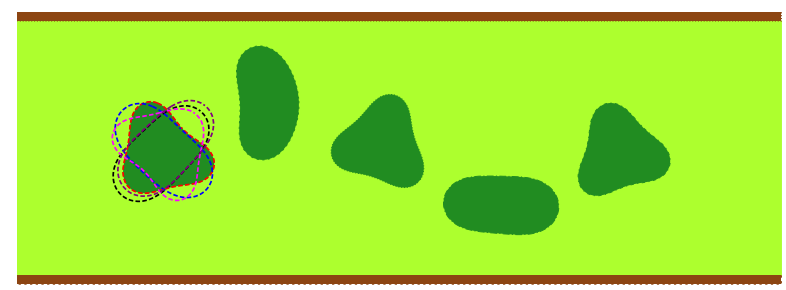

FIG. 4: (Color online) Snapshots of a navigating swimmer over time $\left(W=6 R_{0}\right)$. The dashed profiles show a complete period $T_{s}$ of deformation and then a few shapes are represented over a navigation period $T$ of the order of $50 T_{s}$. $\Gamma=0.085$.

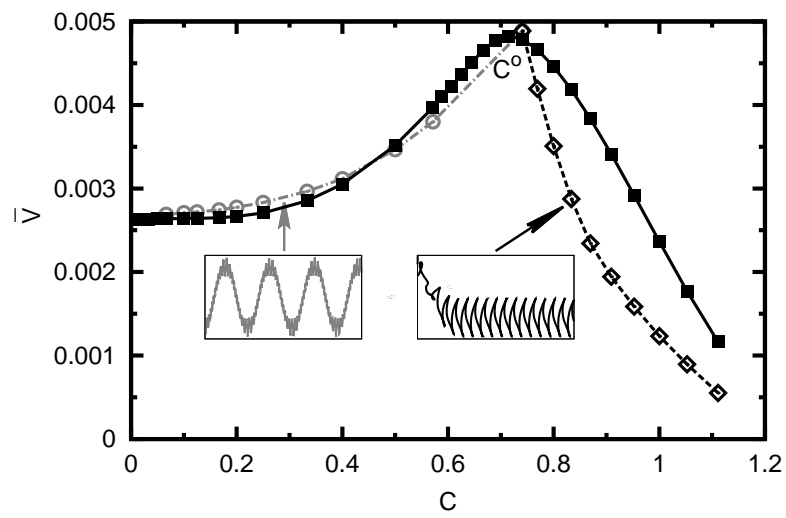

FIG. 5: Time-averaged velocity magnitudes (as a function of confinement $C$ ) of different swimmers $(\Gamma=0.085)$ : migrating along one wall (black diamond dashed line), navigating between two walls (gray circle dotted dashed line) and moving along the channel center (black square solid line). The insets show characteristic trajectories.

puller is observed.

Results: Instability of the central position. The central position after a long time is found to be unstable. The swimmer exhibits at small $C$ (weakly confined regime) a zigzag motion undergoing large amplitude excursions from one wall to the other. We refer to this as a navigating swimmer (NS). Figure 4 shows a snapshot, whereas the insets of Fig. 5 display typical trajectories. Despite this complex motion, the velocity in Fig. [5 behaves with $C$ qualitatively as that of the central swimmer.

The NS trajectory was recently reported 21, 34] in the cases of squirmer and three-bead models and also observed experimentally for paramecium (ciliated motil-

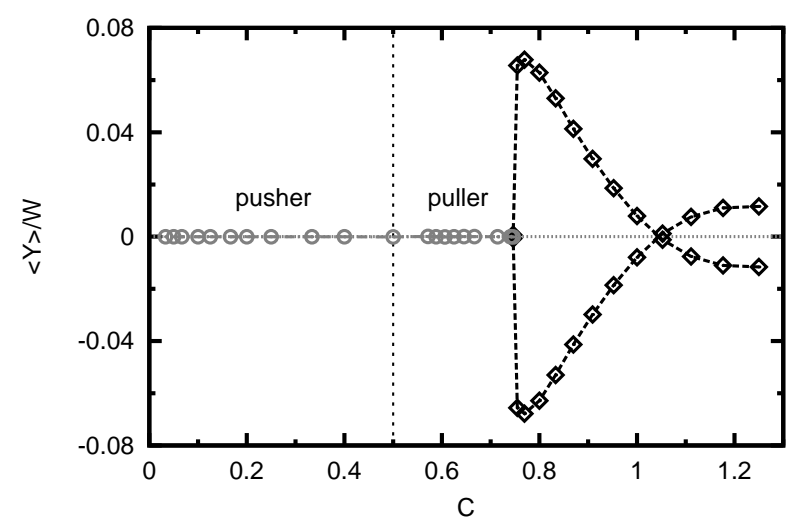

FIG. 6: Average position of the center of mass over a navigation period as a function of confinement $C . \quad \Gamma=0.085$. Circles (diamonds) correspond to the symmetric (asymmetric) motion of the swimmer. The vertical dotted line is the demarcation line between a pusher and puller. Note that a puller can either navigate or move close to either wall. 


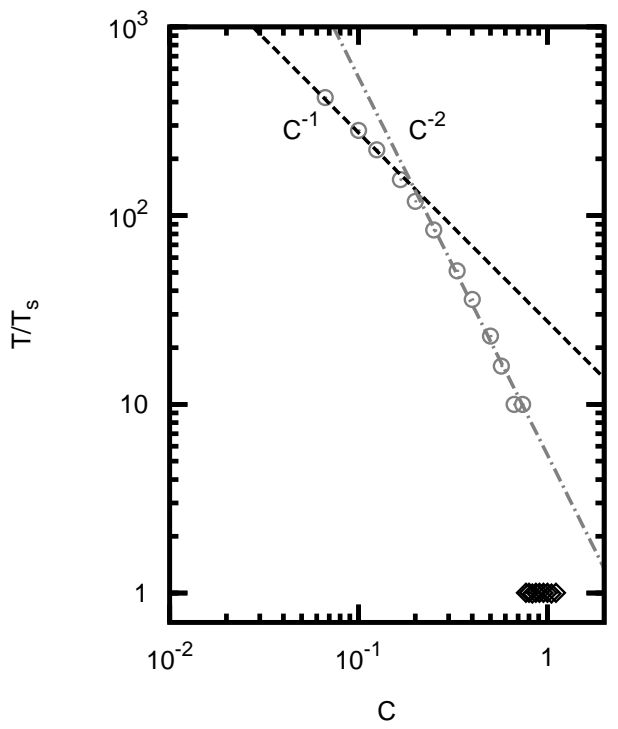

FIG. 7: Period of navigation as a function of confinement $C$. $\Gamma=0.085$. Circles (diamonds) correspond to the symmetric (asymmetric) motion of the swimmer.

ity) in a tube [19] pointing to the genericity of navigation. This instability can be explained analytically (see Ref. [33]).

A remarkable property is that the navigation mode can be adopted both by the pusher and the puller. This is in contrast with nonamoeboid motion [21], where a pusher is found to crash into the wall whereas the puller settles into a straight trajectory. These last two behaviors are also recovered by our simulations, provided the stresslet amplitude is large enough $\left(\Sigma^{2}>>-\bar{V} D S\right.$, where $D$ is the dimensionless force quadrupole strength; see Ref. [33]).

Results: Symmetry-breaking bifurcation. At a critical $C^{*}$ the symmetric excursion of the swimmer becomes unstable and undergoes a bifurcation characterized by the loss of the central symmetry in favor of an asymmetric excursion in the channel, as shown in the trajectories of Fig. [5] (see the supplemental movies in Ref. 33]). Figure 6] shows the average position in $y$ of the center of mass as a function of confinement: a bifurcation diagram. This bifurcation is very abrupt, albeit it is of supercritical nature. Both slightly before and beyond the bifurcation, the swimmer behaves on average as a puller, but still it exhibits two very distinct modes of locomotion: navigation or settling into a quasistraight trajectory (oscillation of the center of mass in this regime is fixed by the amoeboid cycle). This complexity is triggered by the intricate nature of the amoeboid degrees of freedom.

Results: Other force distributions. Including force distributions up to sixth harmonics with various amplitudes leaves the overall picture unchanged, pointing to the generic character of AS. The next step has consisted in linking the nature of the swimmer to its dynamics.
We have monitored a pusher or puller type of swimmer. If $\mathbf{F}=2[\sin (\omega t) \cos (3 \alpha)-(\beta+\cos (\omega t)) \cos (2 \alpha)] \mathbf{n}$, we have a puller; while if $\mathbf{F}=2[(-\beta+\sin (\omega t)) \cos (3 \alpha)-$ $\cos (\omega t) \cos (2 \alpha)] \mathbf{n}$, we have a pusher (with $\beta>0$ ). $\beta$ monitors the strength of the swimmer nature (weak and strong pusher or puller). We found that for a weak enough stresslet, symmetric and asymmetric navigations prevail both for pullers and pushers. For a strong enough stresslet amplitude (for $\beta>\beta_{c} \sim 1$ ), we find that the pusher crashes into the wall, while a puller settles into a straight trajectory. This means that there is a qualitative change of behavior triggered by $\beta$, on which we shall report on systematic study in the future.

Results: Navigation period. The navigation period $T$ exhibits a nontrivial behavior with $C$ (Fig. 7). At small $C$, the period scales as $T \sim C^{-1}$, and as $T \sim C^{-2}$ at intermediate confinement, before attaining a plateau at stronger confinement. To dig into the reasons for this complex behavior, we provide here some heuristic arguments. In the first regime, the NS swims in a straight and monotonous manner towards the next wall. In that regime, the period is limited by the distance traveled by the swimmer of the order $W=2 R / C$. This naturally yields the $C^{-1}$ scaling of Fig. 7 for weak $C$. In the intermediate confinement regime, the magnitude of velocity depends linearly on $C$, so that the period scales as $C^{-2}$ (see also Ref. [33]). After the symmetry-breaking occurs, the NS stays close to one of the two walls (inset of Fig. (5)), and its center of mass oscillates with the intrinsic stroke period $T_{s}$. In this regime, the period is independent of $C$ (diamonds in Fig. 7).

Analytical results. We have first performed a linear stability analysis 33. We find, for small $C$, that the stability of the swimmer is governed by the stresslet sign: For $\Sigma>0$ (pusher) the straight trajectory is unstable, while it is stable otherwise (puller) . For a neutral swimmer the trajectory is marginally stable (the stability eigenvalue $\Omega$, for a perturbation of the form $y \sim e^{\Omega t}$, is purely imaginary). We find that in the intermediate $C$ regime the navigation period behaves as $C^{-2}$. Using a systematic multipole expansion the complex behavior of the velocity as a function of $C$ (at low $C$ ) can be explained [33].

Discussion. We believe that the global features revealed by our study will persist in 3D although extending our work to $3 \mathrm{D}$ simulations will be a challenging task. Besides, in order to better match real cells performing amoeboid swimming (e.g. leukocytes), cytoskeleton dynamics and its relation to force generation will be important ingredients to be included in a $3 \mathrm{D}$ modeling.

C.M., A.F., M.T. and H.W. are grateful to CNES and ESA for financial support. S.R. and P.P. acknowledge financial support from ANR. All the authors acknowledge the French-Taiwanese ORCHID cooperation grant. W.F. H., M.-C.L. and C.M. thank the MoST for a financial support allowing initiation of this project. 
* These authors equally contributed to this work

† Electronic address: salima.rafai@ujf-grenoble.fr

[1] J. Throndsen, Norw. J. Bot. 16, 161 (1969).

[2] N. P. Barry and M. S. Bretscher, Proc. Natl. Acad. Sci. U.S.A. 107, 11376 (2010).

[3] A. J. Baea and E. Bodenschatz, Proc. Natl. Acad. Sci. U.S.A. 107, E167 (2010).

[4] S. Pinner and E. Sahai, J. Micros. 231, 441 (2008).

[5] T. Ohta and T. Ohkuma, Phys. Rev. Lett. 102, 154101 (2009).

[6] T. Hiraiwa, K. Shitaraa, and T. Ohta, Soft Matter 7, 3083 (2011).

[7] A. Shapere and F. Wilczek, Phys. Rev. Lett. 58, 2051 (1987).

[8] J. E. Avron, O. Gat, and O. Kenneth, Phys. Rev. Lett. 93, 186001 (2004).

[9] F. Alouges, A. Desimone, and L. Heltai, Math. Models Methods Appl. Sci. 21, 361 (2011).

[10] J. Loheac, J.-F. Scheid, and M. Tucsnak, Acta Appl. Math. 123, 175 (2013).

[11] A. Vilfan, Phys. Rev. Lett. 109, 128105 (2012).

[12] A. Farutin, S. Rafai, D. K. Dysthe, A. Duperray, P. Peyla, and C. Misbah, Phys. Rev. Lett. 111, 228102 (2013).

[13] T. Lämmermann, B. L. Bader, S. J. Monkley, T. Worbs, R. Wedlich-Soldner, K. Hirsch, M. Keller, R. Forster, D. R. Critchley, R. Fassler, et al., Nature (London) 453, 51 (2008).

[14] X. Garcia, S. Rafaï, and P. Peyla, Phys. Rev. Lett. 110, 138106 (2013).

[15] J. Kessler, Contemp. Phys. 26, 147 (1985).

[16] J. B. McCarthy, S. L. Palm, and L. T. Furcht, J. Cell Biol. 97, 772 (1983).
[17] I. Cantat and C. Misbah, Phys. Rev. Lett. 83, 235 (1999).

[18] B. U. Felderhof, Phys. Fluids 22, 113604 (2010).

[19] S. Jana, S. H. Um, and S. Jung, Phys. Fluids 24, 041901 (2012).

[20] A. Zöttl and H. Stark, Phys. Rev. Lett. 108, 218104 (2012).

[21] L. Zhu, E. Lauga, and L. Brandt, J. Fluid Mech. 726, 011701 (2013).

[22] A. Bilbao, E. Wajnryb, S. A. Vanapalli, and J. Blawzdziewicz, Phys. Fluids 25, 081902 (2013).

[23] R. Ledesma-Aguilar and J. M. Yeomans, Phys. Rev. Lett. 111, 138101 (2013).

[24] A. Acemoglu and S. Yesilyurt, Biophys. J. 106, 1537 (2014).

[25] B. Liu, K. S. Breuer, and T. R. Powers, Phys. Fluids 26, 011701 (2014).

[26] A. Zöttl and H. Stark, Phys. Rev. Lett. 112, 118101 (2014).

[27] F. Z. Temel and S. Yesilyurt, Bioinspiration Biomimetics 726, 016015 (2015).

[28] D. Katz, J . Fluid Mech 64, 33 (2013).

[29] E. Lauga and T. Powers, Rep. Prog. Phys. 72, 096601 (2009).

[30] M. B. Wan, C. J. O. Reichhardt, Z. Nussinov, and C. Reichhardt, Phys. Rev. Lett. 101, 018102 (2008).

[31] M. Thiébaud and C. Misbah, Phys. Rev. E 88, 062707 (2013).

[32] Y. Kim and M.-C. Lai, J. Comp. Phys. 229, 4840 (2010).

[33] See Supplemental Material at [URL will be inserted by publisher] for the theoretical analyses of confined swimming velocity and the relation between the stability of trajectory and the nature of swimmer shown in the paper as well as the movies for two different swimming modes.

[34] A. Najafi, S. S. H. Raad, and R. Yousefi, Phys. Rev. E 88, 045001 (2013). 